\title{
Reconfigurable SiC Embedded Photonic Structures with Self Optical Bias Control
}

\author{
M. Vieira • M. A Vieira • P. Louro • A. Fantoni • V. Silva
}

Received: 8 May 2012 / Accepted: 6 August 2012 /Published online: 13 September 2012

(C) Springer Science+Business Media, LLC 2012

\begin{abstract}
Multilayered heterostructures based on embedded a-Si:H and a-SiC:H p-i-n filters are analyzed from differential voltage design perspective using short- and long-pass filters. The transfer functions characteristics are presented. A numerical simulation is presented to explain the filtering properties of the photonic devices. Several monochromatic pulsed lights, separately (input channels) or in a polychromatic mixture (multiplexed signal) at different bit rates, illuminated the device. Steady-state optical bias is superimposed from the front and the back side. Results show that depending on the wavelength of the external background and impinging side, the device acts either as a short- or a long-pass band filter or as a band-stop filter. Particular attention is given to the amplification coefficient weights, which allow to take into account the wavelength background effects when a band or frequency needs to be filtered or the gate switch, in which optical active filter gates are used to select and filter input signals to specific output ports in wavelength division multiplexing (WDM) communication systems. This nonlinearity provides the possibility for selective removal or addition of wavelengths. A truth table of an encoder that performs 8-to-1 MUX function exemplifies the optoelectronic conversion.
\end{abstract}

M. Vieira $(\triangle) \cdot$ M. A. Vieira $\cdot$ P. Louro $\cdot$ A. Fantoni $\cdot$ V. Silva Electronics Telecommunication and Computer Department, ISEL, R. Conselheiro Emídio Navarro, 1949-014 Lisbon, Portugal

e-mail: mv@isel.ipl.pt

M. Vieira $\cdot$ M. A. Vieira $\cdot$ P. Louro $\cdot$ A. Fantoni $\cdot$ V. Silva

CTS-UNINOVA,

Quinta da Torre, Monte da Caparica,

2829-516 Caparica, Portugal

\section{Vieira}

DEE-FCT-UNL,

Quinta da Torre, Monte da Caparica,

2829-516 Caparica, Portugal
Keywords Photonic active filters · Reconfigurable devices · Numerical simulation $\cdot$ Transfer function characteristics . Encoder-decoder devices

\section{Introduction}

There has been much research on semiconductor devices as elements for optical communication, when a band or frequency needs to be filtered from a wider range of mixed signals [1]. Multilayered structures based on amorphous silicon technology are expected to become reconfigurable to perform wavelength division multiplexing (WDM) optoelectronic logic functions and provide photonic functions such as signal amplification and switching $[2,3]$. They will be a solution in WDM technique for information transmission and decoding in the visible range [4]. The basic operating principle is the exploitation of the physical properties of a nonlinear element to perform a logic function, with the potential to be rapidly biasing tuned. Amplification and amplitude change are two key functionality properties outcome of a balanced interaction between frequency and wavelength of the optical signal and background wavelength and placement within a WDM link. Any change in any of these factors will result in filter readjustments. Here, signal variations with and without front and back backgrounds move electric field action up and down in a known time frame. A numerical simulation support new optoelectronic logic architecture.

\section{Device Configuration and Operation}

Systems that transmit, receive, and/or process intelligence require some form of modulation, which is the deliberate distortion of a carrier to impress intelligence (data) upon it, 
and allow the subsequent recovery of the information. Here, the manipulation of the magnitude is achieved by changing the wavelength (color channels) of the modulated lights and its frequency under appropriated wavelength backgrounds and impinging sides.

The active device consists of a $\mathrm{p}-\mathrm{i}(\mathrm{a}-\mathrm{SiC}: \mathrm{H})-\mathrm{n} / \mathrm{p}-\mathrm{i}(\mathrm{a}-\mathrm{Si}$ : $\mathrm{H})$-n heterostructure with low conductivity doped layers (Fig. 1). The thicknesses and optical gap of the front 'i'$(200 \mathrm{~nm} ; 2.1 \mathrm{eV})$ and back i- $(1,000 \mathrm{~nm} ; 1.8 \mathrm{eV})$ layers are optimized for light absorption in the blue and red ranges, respectively [5]. As a result, both front and back pin structures act as optical filters confining, respectively, the blue and the red optical carriers.

Several monochromatic pulsed lights, separately $\left(\lambda_{\mathrm{R}, \mathrm{G}, \mathrm{B}}\right.$ input channels) or in a polychromatic mixture (multiplexed signal) at different bit rates, illuminated the device from the glass side. Steady-state optical bias with different wavelength is superimposed $(400-800 \mathrm{~nm})$ from the front or from the back sides and the generated photocurrent measured at $-8 \mathrm{~V}$. The device operates within the visible range using as input color channels (data) with the wave square modulated light (external regulation of frequency and intensity) supplied by a red $\left(R, 626 \mathrm{~nm} ; 51 \mu \mathrm{W} / \mathrm{cm}^{2}\right)$, a green $(G, 524 \mathrm{~nm} ; 73 \mu \mathrm{W} /$ $\left.\mathrm{cm}^{2}\right)$, and a blue $\left(B, 470 \mathrm{~nm} ; 115 \mu \mathrm{W} / \mathrm{cm}^{2}\right)$ LED. Additionally, steady-state red, green, blue, and violet (background) was superimposed by different LEDs $\left(R, 625 \mu \mathrm{W} / \mathrm{cm}^{2} ; G\right.$, $\left.515 \mu \mathrm{W} / \mathrm{cm}^{2} ; B, 400 \mu \mathrm{W} / \mathrm{cm}^{2} ; V, 2,800 \mu \mathrm{W} / \mathrm{cm}^{2}\right)$.

\section{Numerical Simulation}

\section{Input Parameters}

In order to understand the light filtering properties of the device, under different electrical and optical bias conditions, a simulation program ASCA-2D [6] was used having as input parameters the experimental data. For the a-SiC:H and a-Si:H absorbers, an optical band gap of 2.1 and $1.8 \mathrm{eV}$ and a thickness of 200 and 1,000 nm were chosen, respectively. The doping level was adjusted in order to obtain approximately the same conductivity of the typical thin film layers [7].

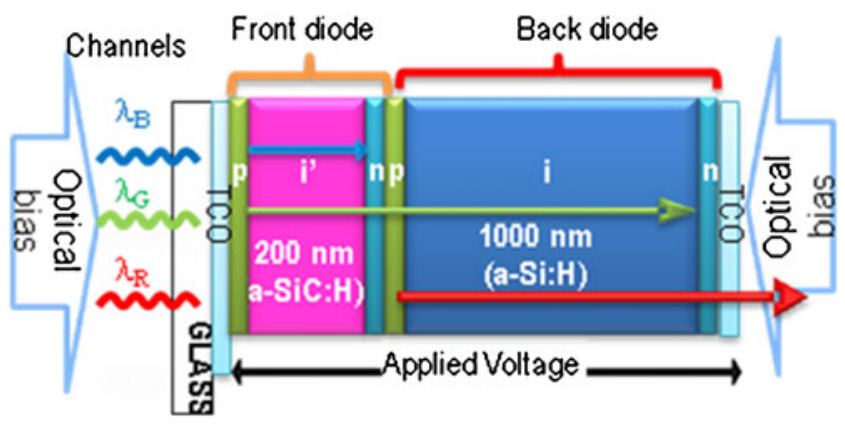

Fig. 1 Device configuration and operation
In Fig. 2, the simulated spectral sensitivity of the front (pi'-n) and back (p-i-n) diodes are compared with the normalized experimental sensitivity of the individual front and back diodes. A good fit between simulated and experimental results was achieved for the individual optimized diodes. As a result, both front and back pin structures act separately as optical filters confining, respectively, the blue and the red optical carriers to their active absorption areas.

\section{Background Wavelength}

Under negative applied voltage, in Fig. 3, it is reported in the generation rate (a) and the electric field (b) profiles under different front wavelengths backgrounds. Results show that the balance between the electrical field adjustments due to the nonuniform absorption throughout the structure depends on the generation profile at each background wavelength. When an external electrical or optical bias is applied to a double pin structure, its main influence is in the field distribution within the less photo excited sub-cell. In comparison with thermodynamic equilibrium conditions (no background), the electrical field under illumination is lowered in the most absorbing cell (self forward bias effect), while the less absorbing reacts by assuming a reverse bias configuration (self reverse bias effect) [8]. So, the shallow penetration of the blue photons into the front diode, the deep penetration of the red photons exclusively into the back diode, or the decay of the green absorption across both controls the sensor behavior.

Both the front and the back diodes are optically and electrically in series. Under steady-state irradiation, to sustain the current across the device, the current at the less absorbing diode has to be adjusted through an increase of the electrical field and thus it becomes reverse biased. The superposition of a color channel will affect locally this field. Under red background (Fig. 3b), the blue channel increases the field intensity in the front diode and even reverses it at the internal interface increasing carrier collection. The red and the green channels change the field in an opposite way.

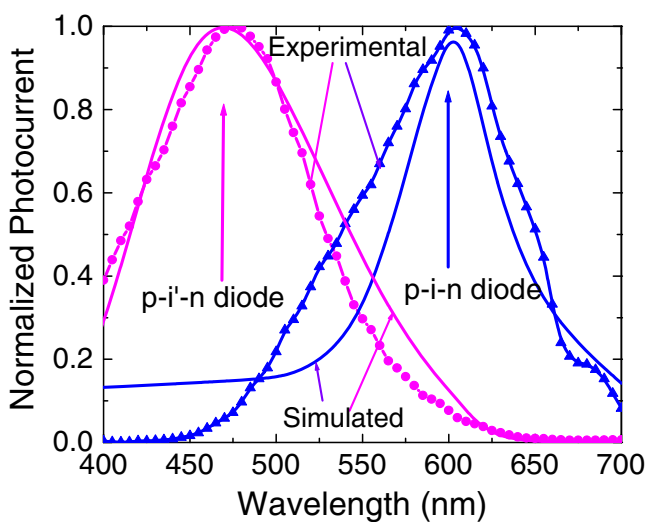

Fig. 2 Comparison between simulated (lines) and experimental (symbols) normalized front (p-i'-n) and back (p-i-n) diodes 
Fig. 3 Generation (a) and electric field (b) profiles within the $\mathrm{p}-\mathrm{i}$ '-n/p-i-n tandem structure under different wavelengths backgrounds $\left(\lambda_{\mathrm{R}, \mathrm{G}, \mathrm{B}}\right)$ and without it

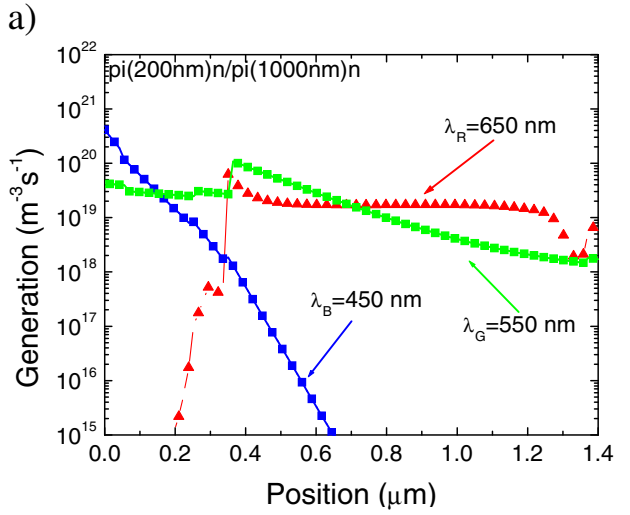

b)

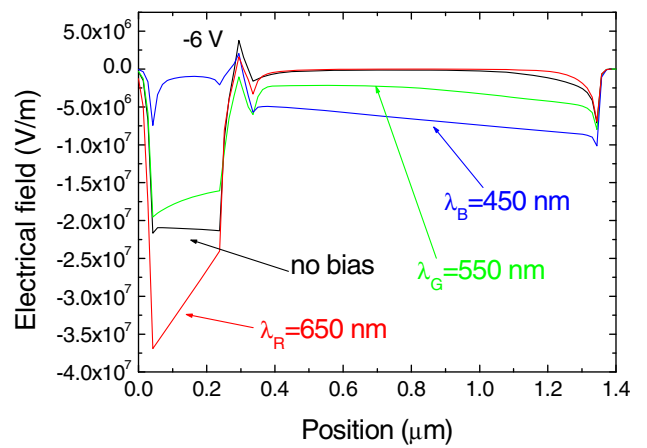

Front and Back Background

Based on the optimized input parameters, Fig. 4 reports the simulated generation (solid lines) and recombination (dash lines) rate profiles of the red, green, and blue input channels, under front (a) and back (b) violet optical bias.

Results show that the depth of light penetration within the device depends on both the wavelength of the input channels and background side. Under violet irradiation, the profile is strongly influenced by the choice of the device side for the light incidence. Under front irradiation, the generation in the front diode increases mainly for the long wavelengths channels (green and red) due to the contribution of the background light that generates carriers exclusively in the front diode. Under back irradiation, the generation in the back photodiode increases for short/medium input wavelengths due to the light penetration depth of the violet light across the bottom of the a-Si:H intrinsic layer.

The spectral sensitivity of the device results from the interaction of the electric field under applied optical bias (red, green, blue, and violet) and the transient electric field induced by the input channels (red, green, and blue). This interaction results in electric field lines that guides the photocarrriers generated by the input channels.

In Fig. 5, the simulated electric field profiles under front and back violet background when the red, green and blue channels are ON and OFF are displayed. Results show that the flow rate of the carriers through those field lines towards the output depends not only on the on/off state of the channels but also on the irradiation side.

The front violet background (Fig. 4a) is absorbed at the surface of the front diode, increasing the electric field at the back diode, where the red and part of the green channels generate optical carriers. Under back irradiation, the small absorption depth of the violet photons across the back diode (Fig. 4b) quenches the electric field there and enhances it in the front one where the blue and part of the green channels generate the photocarriers. A reversal at the internal interface is also observed leading to different charge accumulation regions under the front and back violet irradiation.

\section{Transfer Function Characteristics}

\section{Spectral Sensitivity}

The transfer function magnitude (or gain) allows determining the ability of the optical filter to distinguish between signals at different wavelengths. The spectral sensitivity was tested through spectral response measurements under different frequencies, with and without steady-state optical bias applied either from the front or back side.
Fig. 4 Generation rate profile of the device under: a front and b back steady-state violet illumination a)

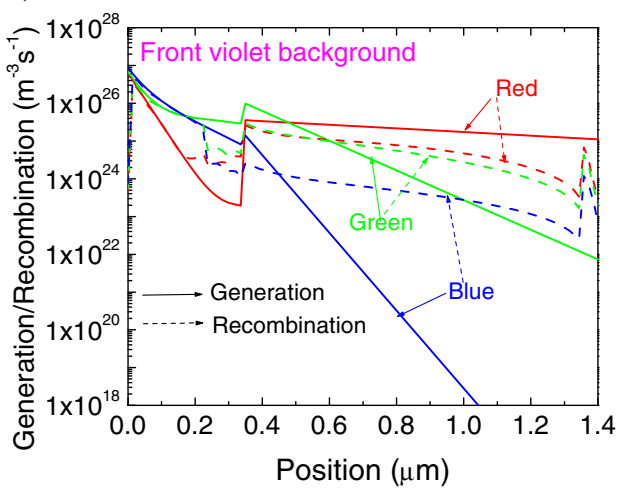

b)

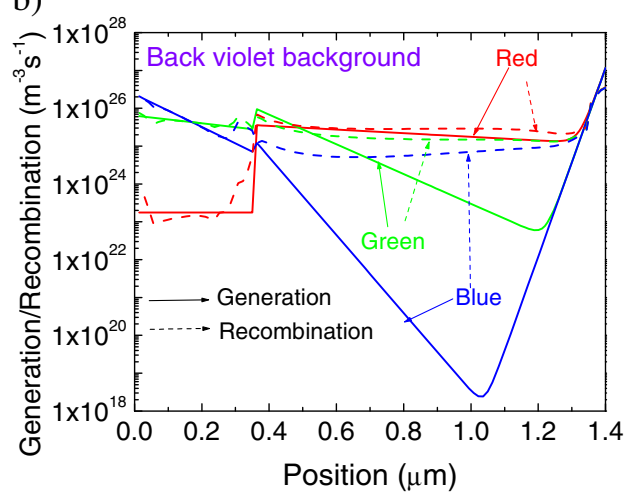


Fig. 5 Simulated electric field profile under front and back violet background when the red, green, and blue channels are $\mathrm{ON}$ and $\mathrm{OFF}$

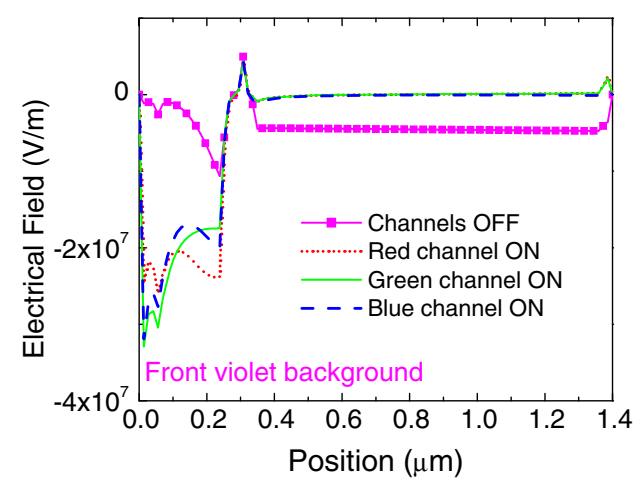

In Fig. 6, at $500 \mathrm{~Hz}$, the spectral photocurrent (symbols) is displayed under red, green, blue, and violet backgrounds and without it. In Fig. 6a, the steady-state optical bias was applied from the front side and in Fig. $6 \mathrm{~b}$ from the back side. For comparison the normalized spectral photocurrent for the front, $\mathrm{p}-\mathrm{i}^{\prime}-\mathrm{n}$, and the back, $\mathrm{p}-\mathrm{i}-\mathrm{n}$, photodiodes (dash lines) are superimposed.

Data show that the front and back building blocks, separately, present the typical response of single $\mathrm{p}-\mathrm{i}-\mathrm{n}$ cells with intrinsic layers based on a-SiC:H or a-Si:H materials, respectively. The front diode cuts the wavelengths higher than $550 \mathrm{~nm}$ while the back one rejects the ones lower than
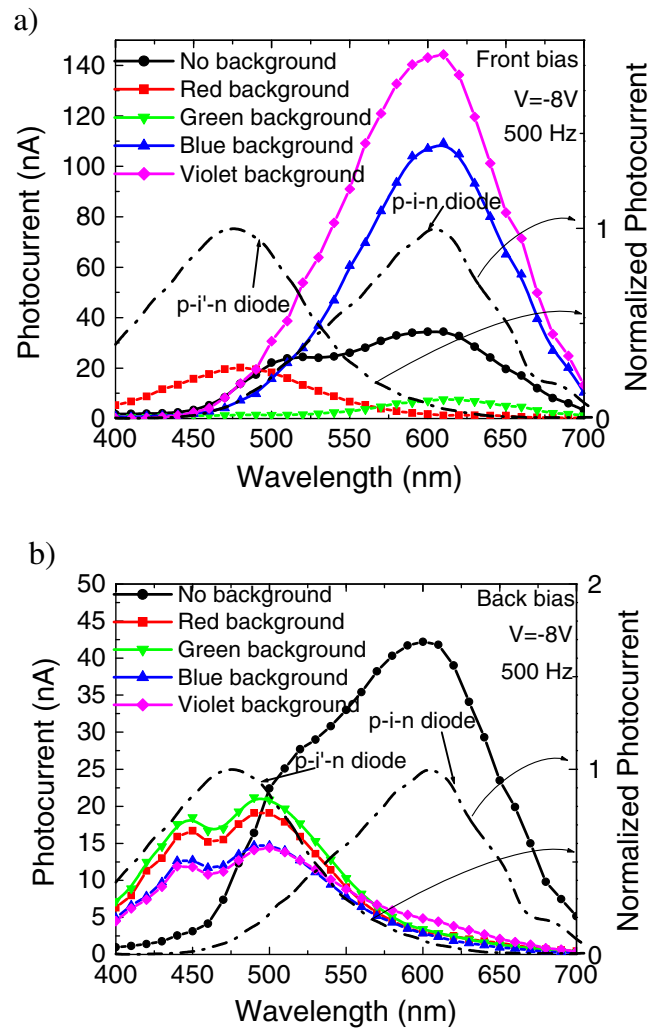

Fig. 6 Photocurrent without and with front (a) and back (b) backgrounds. The current of the individual photodiodes are superimposed (dash lines)
$500 \mathrm{~nm}$. The overall device presents an enlarged sensitivity when compared with the individual ones.

Under front irradiation, the sensitivity is much higher than under back irradiation. Under front irradiation (Fig. 6a), the violet background amplifies the spectral sensitivity in the visible range while the blue optical bias only enhances the spectral sensitivity in the long wavelength range $(>550 \mathrm{~nm})$ and quenches it in the others. Under red bias, the photocurrent is strongly enhanced at short wavelengths and disappears for wavelengths higher than $550 \mathrm{~nm}$. Under green, the sensitivity is strongly reduced in all the visible spectra. In Fig. 6b, whatever the wavelength of the backgrounds, the back irradiation strongly quenches the sensitivity in the long wavelength range $(>550 \mathrm{~nm})$ and enhances the short wavelength range. So, back irradiation tunes the front diode while front irradiation, depending on the wavelength used, tunes the back one.

\section{Optical Bias-Controlled Filters}

In Fig. 7, the spectral gain, defined as the ratio between the spectral photocurrents under red $\left(\alpha^{\mathrm{R}}\right)$, green $\left(\alpha^{\mathrm{G}}\right)$, blue $\left(\alpha^{\mathrm{B}}\right)$, and violet $\left(\alpha^{\mathrm{V}}\right)$ illumination and without it, is plotted at 500 and 3,500 Hz. The optical bias is applied from the front side in Fig. $7 \mathrm{a}$ and from the back side in Fig. $7 \mathrm{~b}$.

Under front bias and red irradiation, the gain is high at short wavelengths and strongly lowers for wavelengths higher than $500 \mathrm{~nm}$, acting as a short-pass filter. Under green background and high frequencies, the device behaves as a band-stop filter that screens out the medium wavelength range (green) enhancing only the photocurrent for wavelengths outside of that range. Under blue and violet light, the device works as a long-pass filter for wavelengths higher than $550 \mathrm{~nm}$, blocking the shorter wavelengths. Back light, whatever the frequency, leads to a short-pass filter performance. Results show that by combining the background wavelengths and the irradiation side the short-, medium-, and long-spectral region can be sequentially tuned. In Fig. 8, the gains at fixed wavelengths: $470 \mathrm{~nm}$ (blue channel), $526 \mathrm{~nm}$ (green channel), $624 \mathrm{~nm}$ (red channel), under violet front (symbols) and back (lines) backgrounds, are plotted as a function of the frequency. 
Fig. 7 Spectral gain under red $\left(\alpha^{\mathrm{R}}\right)$, green $\left(\alpha^{\mathrm{G}}\right)$, and blue $\left(\alpha^{\mathrm{B}}\right)$ optical bias, applied from the front (a) and the back (b) sides at different frequencies

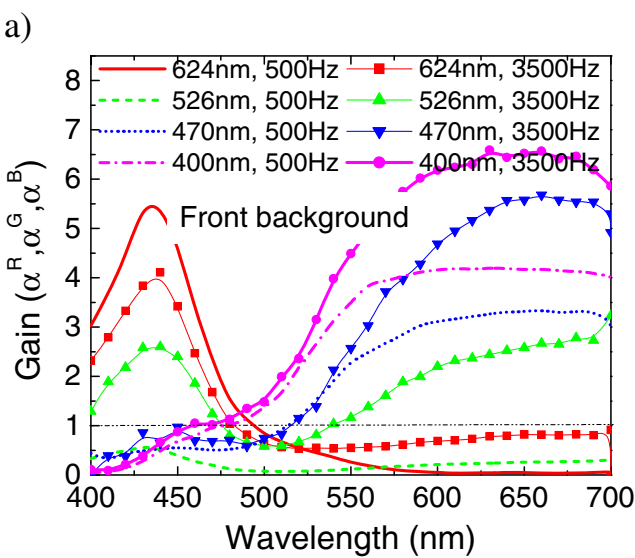

b)

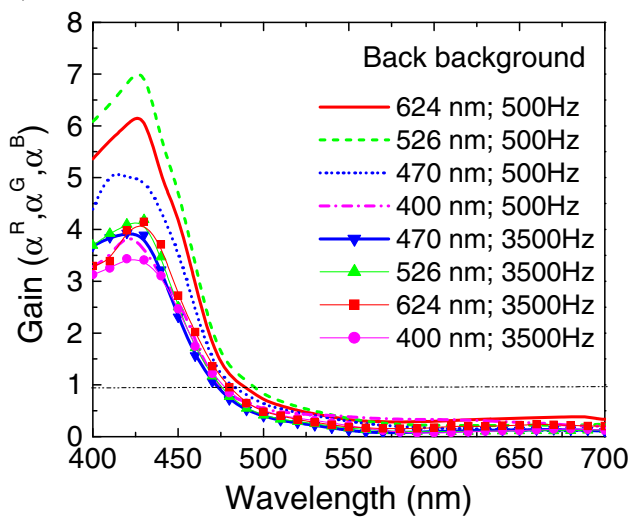

Results show that, whatever the frequency, the device acts as an active long-pass filter under front irradiation and a low-pass filter under back irradiation. No matter what the irradiation side, the blue and green channel gain does not depend on the frequencies, while the red one increases under front illumination and is strongly reduced under back light.

From Figs. 7 and 8, under front bias control, the gain is higher than the unity for the long wavelengths resulting in an amplification of the green and red spectral channels. Back violet irradiation only amplifies the short wavelength range (blue channel) and quenches the others.

\section{Encoder and Decoder Device}

Violet Background-Controllable Wavelength Selective Optical Switching

To analyze the device under information-modulated wave and uniform irradiation, three monochromatic pulsed lights separately (red, green and blue input channels, Fig. 9a) or combined (multiplexed signal, Fig. 9b) illuminated the device at 6,000 bps. Steady-state violet optical bias was superimposed separately from the front (solid lines, $\operatorname{pin}_{1}$ ) and the

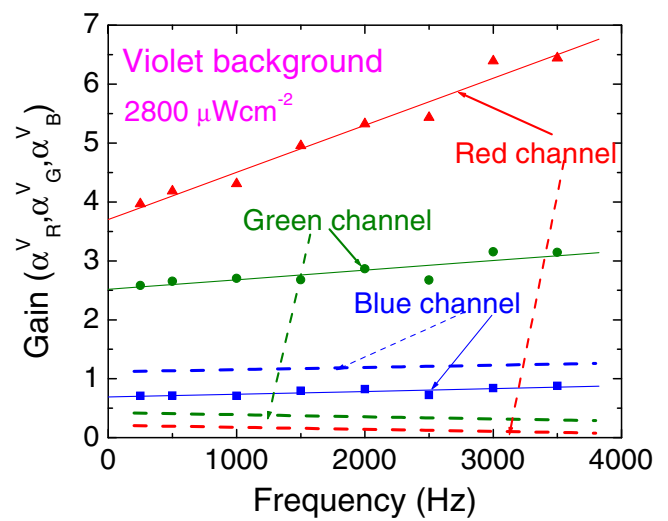

Fig. 8 Input front (symbols) and back (lines) channel gains $\left(\alpha_{\mathrm{R}}{ }^{\mathrm{V}}, \alpha_{\mathrm{G}} \mathrm{V}\right.$, and $\left.\alpha_{\mathrm{B}} \mathrm{V}\right)$, as a function of the frequency back (dash lines, $\operatorname{pin}_{2}$ ) sides and the photocurrent generated measured at $-8 \mathrm{~V}$.

The transient signals were normalized to their values without background in Fig. 9a. In Fig. 9b, the normalized multiplexed signal under front (symbols) and back (lines) violet irradiation is displayed. On the top of the figure, the signals used to drive the input channels are shown to guide the eyes into the ON/OFF channel states.

Even under transient conditions, the front background presents a nonlinear dependence on the wavelength. It enhances mainly the light-to-dark sensitivity in the medium-long
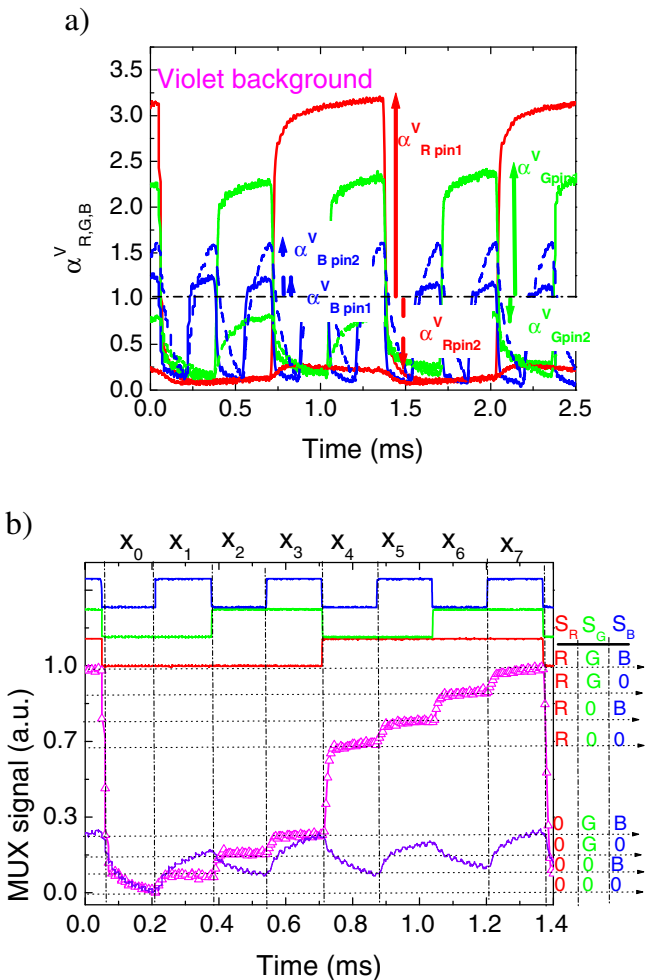

Fig. 9 a Normalized red, green, and blue transient signals at $-8 \mathrm{~V}$ with violet $(400 \mathrm{~nm})$ steady-state optical bias applied from the front side $\left(\alpha_{\text {pin1 }}\right)$ and from the back side $\left(\alpha_{\text {pin2 }}\right)$. b Multiplexed output signal under front (symbols) and back (line) violet irradiation 


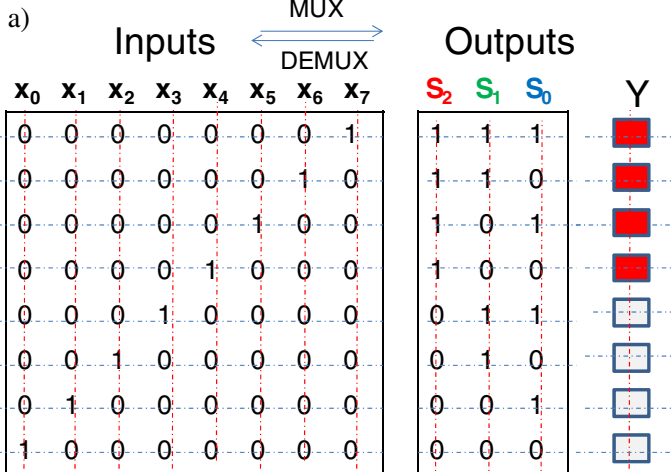

b)

$$
\text { Inputs } \stackrel{\text { MUX }}{\longleftrightarrow \text { DEMUX }} \text { Outputs }
$$

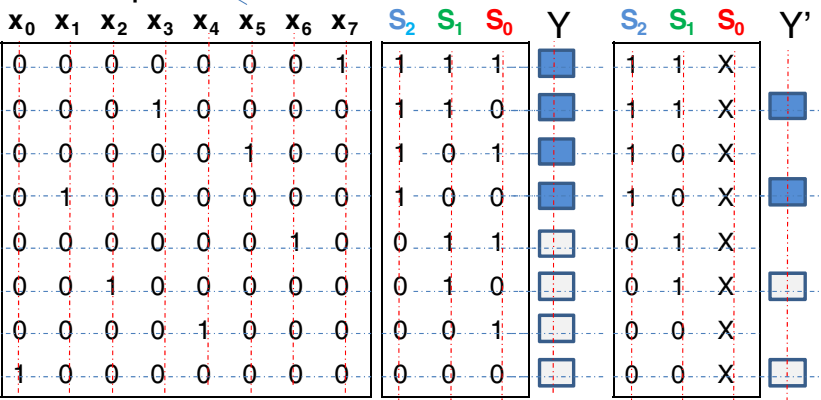

Fig. 10 Truth tables of the encoders that perform 8-to-1 multiplexer (MUX) function, under front (a) and back (b) violet irradiations (multiplication sign " $\times$ " means "not significant")

wavelength ranges. Violet radiation is absorbed at the top of the front diode, increasing the electric field at the least absorbing cell (see Fig. 5), the back diode. So the collection is strongly enhanced $\left(\alpha_{\text {Gpin1 }} \mathrm{V}=2.2, \alpha_{\text {Rpin1 }}{ }^{\mathrm{V}}=3.1\right)$ while the blue collection stays near its dark value $\left(\alpha_{\mathrm{Bpin} 1}{ }^{\mathrm{V}}=1.1\right)$. Under back irradiation, the small absorption depth of the violet photons across the back diode quenches the electric field, and so, the red collection almost disappears $\left(\alpha_{\mathrm{Rpin} 2}{ }^{\mathrm{V}}=0.2\right)$. Blue channel is absorbed across the front diode where the electric field is enhanced resulting in an increase collection of the blue channel $\left(\alpha_{\text {Bpin1 }}{ }^{V}=1.6\right)$. Since the green channel is absorbed across front and back diodes, its collection is balanced by the increased collection in the front diode and its reduction at the back one $\left(\alpha_{\mathrm{Gpin} 2} \mathrm{~V}=0.7\right)$.

\section{Optoelectronic Conversion}

For an optoelectronic digital capture system, optoelectronic conversion is the relationship between the optical input levels and the corresponding digital output levels. We use an 8-bit priority encoder as an example to illustrate how a logic function is mapped onto the proposed directed-logic circuit. An encoder has a number of inputs, only one of which is in the 1 state, and an N-bit code is generated, depending upon which of the inputs is excited.

The truth tables of both encoders of Fig. 9 that perform 8to-1 MUX function are also shown in Fig. 10. In the inputs $\left(x_{0} \ldots x_{7}\right)$, the index of each bit is related to the first (highest) nonzero logic input. Here, the MUX device selects, through the violet background, one of the eight input logic signals and sends it to the output $\left(y=x_{S}\right)$. The output is a 3-bit $\left[S_{2} S_{1} S_{0}\right]$ binary RGB number that may identify one of eight possible inputs.

To understand this mapping, in Fig. 9, for the input $x_{7}$ and output $S_{2}$, the first nonzero logic input is $7\left(2^{2}+2^{1}+2^{0}\right)$, which corresponds an output [111]. Those OR gates are expressed, respectively, as $S_{2}=x_{7}+x_{6}+x_{5}+x_{4}$ under front irradiation (Fig. 10a) and $S_{2}=x_{7}+x_{3}+x_{5}+x_{1}$ under back light (Fig. 10b).

Violet irradiation is applied from the front $\left(\operatorname{pin}_{1}\right.$; $\alpha_{\text {Rpin } 1} \mathrm{v}_{>>1}, \alpha_{\text {Gpin } 1} \mathrm{v}_{>}>1$, and $\left.\alpha_{\text {Bpin }}{ }^{\mathrm{V}} \sim 1\right)$ and back $\left(\operatorname{pin}_{2}\right.$; $\alpha_{\text {Rpin2 }} \mathrm{V}<<1, \alpha_{\text {Gin2 }}{ }^{\mathrm{V}}<1$, and $\alpha_{\text {Bpin2 }}{ }^{\mathrm{v}}>1$ ) sides (see Figs. 8 and 9a). Under front irradiation (Fig. 9b), the $2^{3}$ levels can be grouped into two main classes due to the high amplification of the red channel. The upper four levels are ascribed to the presence of the red channel $\mathrm{ON}$, and the lower four to its absence, allowing the red channel decoder (4-to-1 multiplexer; long-pass filter function). Since under front irradiation, the green channel is amplified, the two highest levels, in both classes, are ascribed to the presence of the green channel and the two lower ones to its lack.

Under back irradiation, the blue channel is enhanced; the green reduced and the red almost suppressed. The encoded multiplexed signal has the eight sublevels grouped also into two main levels, the higher where the blue channel is ON and the lower where it is OFF (4-to-1 multiplexer; shortpass filter function). In each main level, the four existent
Fig. 11 MUX signal under front and back irradiation. On the top, the DEMUX signals obtained using the decoder algorithm is displayed as well as the binary bit sequences
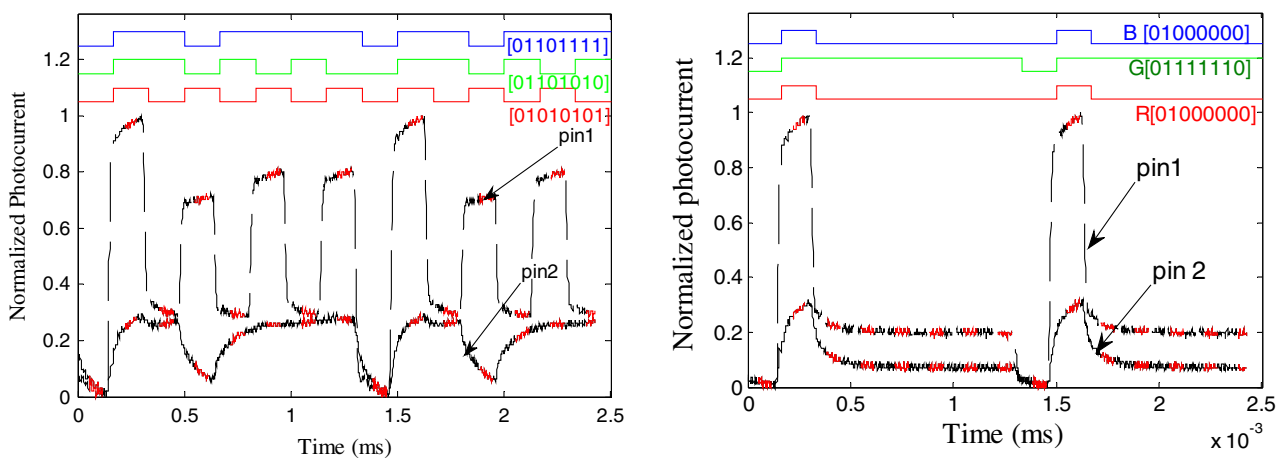
sublevels are grouped in two classes, with and without the channel green ON. Each of those sublevels split into two near ones, attributed to the presence or absence of the red channel. If we consider this red output bit "not significant," only four separate levels $\left(2^{2}\right)$ are considered and the logic MUX function is converted into a logic filter function. The blue channel is then decoded.

Like regular binary numbers, the binary RGB code is an arithmetic code and so it is weighted, i.e., there is specific weights assigned to each bit position. Under front violet irradiation, the most significant digit, the left most bit, in the RGB code is the red $\left(\alpha_{\text {Rpin1 }} \mathrm{V}_{>>1}\right)$. Going from the left to right, the next is the green $\left(\alpha_{\text {Gpin1 } 1} \mathrm{v}_{>}\right)$and the last is the blue $\left(\alpha_{\text {Bpin1 }}{ }^{\mathrm{V}} \sim 1\right)$. Under back violet irradiation, the left most bit is the blue. Going from the left to right, the next is the green and last the red. So, the correspondence between the outputs $S_{2}$, $S_{1}$, and $S_{0}$, in Fig. 10, and the on/off state of the input channels $S_{\mathrm{R}}, S_{\mathrm{G}}$, and $S_{\mathrm{B}}$ in Fig. 9 is obvious.

We have used this simple algorithm to decode the multiplex signal. The results are displayed in Fig. 11 for two different bit sequences. An excellent fit was obtained.

Results show that the pi'npin multilayered structure becomes reconfigurable under front and back irradiation. They perform WDM optoelectronic logic functions providing photonic functions such as signal amplification, filtering, and switching. So, by means of optical control applied to the front or back diodes, the photonic function is modified from a long- to a short-pass filter, giving a step reconfiguration of the device.

\section{Conclusions}

Tandem a-SiC:H pi'n/pin active filters are analyzed under different front and back optical bias wavelengths and frequencies. Results show that the output waveform presents a nonlinear amplitude-dependent response to the wavelengths of the input channels. Under controlled wavelength backgrounds, the light-to-dark sensitivity can be enhanced in a specific wavelength range and quenched in the others, tuning a specific band. Depending on the wavelength of the external background, the device acts either as a short- or a long-pass band filter or as a band-stop filter.

An optoelectronic numerical simulation supports the experimental data and gives insight into the physics of the device. A truth table of an encoder that performs 8-to-1 multiplexer (MUX) function is presented.

Acknowledgments This work was supported by FCT (CTS multi annual funding) through the PIDDAC Program funds and PTDC/EEAELC/111854/2009 and PTDC/EEA-ELC/120539/2010.

\section{References}

1. Connelly MJ (2002) Semiconductor optical amplifiers. Boston: Springer, ISBN 978-0-7923-7657-6

2. Petit C, Blaser M (2006) Workshop on optical components for broadband communication. Fonjallaz P-Y, Pearsall T. P. (ed.), Proc. of SPIE. vol. 6350, 63500I

3. Ibrahim S, Luo LW, Djordjevic SS, Poitras CB, Zhou I, Fontaine NK, Guan B, Ding Z, Okamoto K, Lipson M and Yoo SJB (21 Mar 2010) paper OWJ5. Optical Fiber Communications Conference, OSA/OFC/NFOEC, San Diego

4. Randel S, Koonen AMJ, Lee SCJ, Breyer F, Garcia Larrode M, Yang J, Ng'Oma A, Rijckenberg GJ, Boom HPA (2007). ECOC 07 (Th 4.1.4). Berlin, Germany, pp. 1-4

5. Vieira M, Louro P, Fernandes M, Vieira MA, Fantoni A, Costa J (2011), Advances in Photodiodes, Gian Franco Dalla Betta (ed.), ISBN: 978-953-307-163-3, InTech, Chap.19, pp:403-425

6. Fantoni A, Vieira M, Martins R (1999) Math Comput Simul 49:381-401

7. Vieira M, Fantoni A, Fernandes M, Louro P, Lavareda G, Carvalho CN (2009) Journal of Nanoscience and Nanotechnology 9(7):4022-4027

8. Vieira MA, Vieira M, Costa J, Louro P, Fernandes M, Fantoni A (2010) Sensors \& Transducers Journal 9:96-120 